\title{
State of the Art - Rectal Cancer Surgery
}

\author{
Andreas Bogner Johanna Kirchberg Jürgen Weitz Johannes Fritzmann \\ Department of General, Visceral, Thoracic and Vascular Surgery, University Hospital Carl Gustav Carus, TU Dresden, \\ Dresden, Germany
}

Keywords

Rectal cancer - Total mesorectal excision .

Abdominoperineal resection - Multimodal treatment

\section{Abstract}

Background: In an aging society, the incidence and relevance of rectal cancer as one of the most frequent gastrointestinal cancers gains in importance. Excellent surgery and up-to-date multimodal treatments are essential for adequate oncological results and good quality of life. Summary: In this review, we describe modern developments in rectal cancer surgery and its embedment in modern multimodal therapy concepts. Key Message: Distinguished interdisciplinary cooperation combined with an outstanding surgical expertise is the basic requirement for an optimal treatment of rectal cancer. Thus, high standards of oncological outcome and patient's quality of life can be achieved.

(c) 2019 S. Karger AG, Basel

\section{Introduction}

Colorectal cancer is the third most common tumor entity in Germany with an incidence of about 62,000 new diagnosed carcinomas every year. About one third is located in the rectum ( 25,000/year). Although new surgical approaches and multimodal therapy strategies including radio- and chemotherapy improved during the last three decades, mortality is still amongst the second (male) and third (female) frequent causes of cancer-related death in Germany [1].

\section{KARGER}

(C) 2019 S. Karger AG, Basel
Due to its localization within the rectum, rectal cancers are divided into tumors of the lower $(0-6 \mathrm{~cm})$, middle $(6-12 \mathrm{~cm})$, and upper $(12-16 \mathrm{~cm})$ third, assessed by rigid rectoscopy measured from the anal verge to the lower tumor margin. This classification is essential for the surgical strategy [2].

\section{Development of Rectal Surgery: From APR to TME}

In 1906, Miles established the basis for modern rectal cancer surgery introducing the radical "abdominoperineal resection" (APR) [3]. This procedure includes a conic removal of the rectum including the anus and sphincter. The APR results in creation of a permanent colostomy, which means, especially for younger patients, an enormous discomfort. Throughout the twentieth century, the focus shifted toward sphincter-preserving surgical techniques and the "anterior resection" (AR) became the standard treatment for mid and high rectal cancer [4]. This therapy was limited by high rates of local recurrence of about $20 \%$ [5].

The introduction of the total mesorectal excision (TME) in 1982 by Heald et al. [6] revolutionized the understanding and success of rectal cancer surgery. This radical approach enables the complete resection of the lymphatic tissue up to the plane between the mesorectum and the presacral fascia (Healds "Holy Plane") (Fig. 1, 2). Dissection within this avascular plane facilitated surgery and in parallel allows nerve- and vessel-preserving operations. This procedure, including central vessel ligation, became the gold standard of oncologic rectal surgery for tumors of the lower and middle third of the rectum. For 


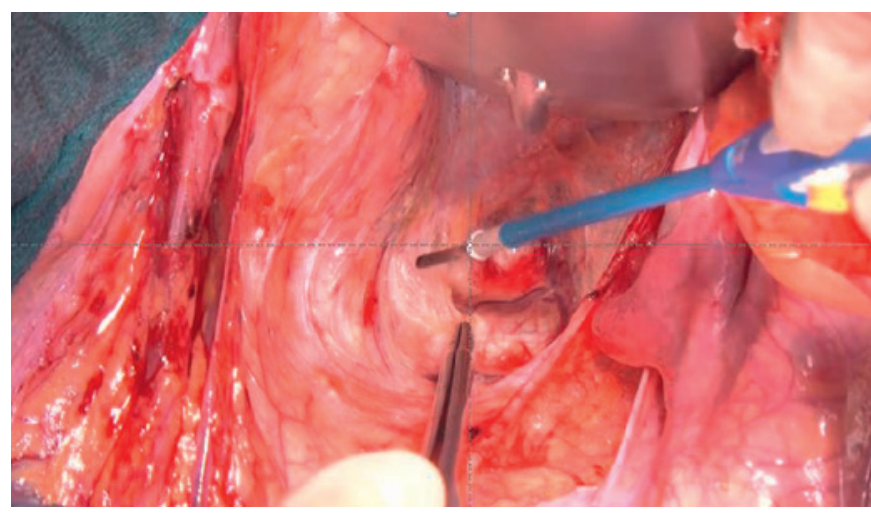

Fig. 1. Open TME.

carcinomas located in the upper third, a partial mesorectal excision with a safety distance of at least $5.0 \mathrm{~cm}$ to the lower tumor margin is recommended in the German S3 guideline [2].

The precise performance of TME within the anatomical borders is essential. Especially the avoiding of "coning" in case of partial mesorectal excision for high rectal cancers is important to ensure resection of the mesorectum in a vertical plane parallel to the intestine in order not to leave lymphatic tissue behind.

Therefore, an exact and sharp preparation along the embryological, anatomical borders is mandatory to dissect the mesorectum from its parietal planes (endopelvic fascia, parietal fascia, Denonvillier's fascia, Waldeyer's fascia). To secure a postoperative adequate sexual, ejaculation, and bladder function, the surgical skills of preserving the autonomous nerves (Nn. hypogastrici, plexus hypogastrici inferior and superior) are compulsive for every rectal surgeon. In terms of quality assurance, the rate of injury of the so-called "holy plane" should not exceed 5\% in sphincter-sparing resection and $10 \%$ in $\operatorname{APR}[7,8]$. The circumferential resection margin (CRM) to the tumor should also be negative. It has been described that a CRM below $1 \mathrm{~mm}$ that can be predicted by pretherapeutic MRI and should be assessed by pathology leads to higher local recurrence rates. In case of infiltration of surrounding tissue (sacral bone, prostate, seminal vesicle, uterus, vagina, etc.), an en-bloc resection of those adherent organs is mandatory, i.e., conduction of a multivisceral resection [9]. Since the broad introduction of TME, the rate of local recurrence lowered from partially $>50 \%$ to under $10 \%$ and consequently, overall survival improved strongly $[10$, 11]. The experience of the surgeon is of essential significance for the oncological outcome. A Swedish and Dutch group could detect a direct correlation of surgical experience, number of operations, and overall survival. It has been shown that intensive training under experienced supervision can positively influence patient's outcome [12,

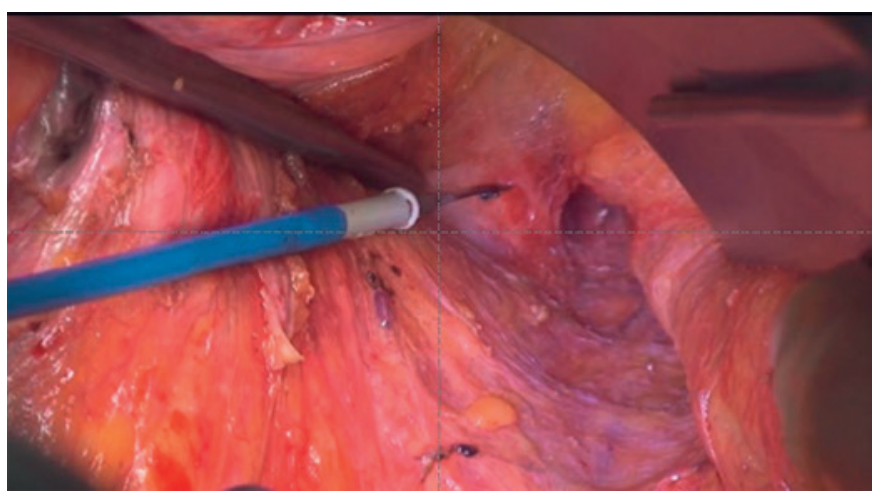

Fig. 2. TME with pelvic nerve plexus.

13]. Therefore, surgeons should make every effort to optimize the quality of surgery, as this is the factor that is of greatest impact for patient outcome and it has been well documented that experience and quality of surgery directly translate into better survival.

In addition to survival, the preservation of the anal sphincter plays a central role for many patients wanting to keep their continence. During the last 3 decades since the introduction of TME surgery, there has been special focus on sphincter-preserving operations. Low-grade tumors with good or medium differentiation in the lower third of the rectum can be resected by keeping a safety margin of 1-2 cm; in case of high-grade tumors, this margin should be wider [14, 15]. After neoadjuvant radiochemotherapy, in order to avoid APR, a distal resection margin of $0.5 \mathrm{~cm}$ can be acceptable when using intraoperative, pathological assessment of tumor-free margins [2]. When evaluating the patient for sphincter-preserving surgery in ultra-low rectal cancer, age, patients' medical history, preoperative sphincter function, and social situation should be taken into consideration. A permanent colonic stoma can sometimes be more convenient than a malfunctioning anal sphincter. If possible, the internal anal sphincter muscle can be partially or completely removed, i.e., an intersphincteric rectal resection can be performed, with results of complete continence in over $50 \%$ of cases combined with acceptable oncologic outcomes $[16,17]$.

After rectal resection, the reservoir function of the ampulla is limited with high rates of urge incontinence. Many efforts have been made to reduce this phenomenon by the development of diverse types of alternative reservoirs. Upon those, transverse coloplasty and J-pouch are the widely used alternatives [18]. In addition, the use of linear and circular stapling devices helped to reduce the rate of anastomotic insufficiency [19] (Fig. 3).

In case of tumor infiltration of the anal channel, the external anal sphincter, or levator, a zylindric APR, in- 


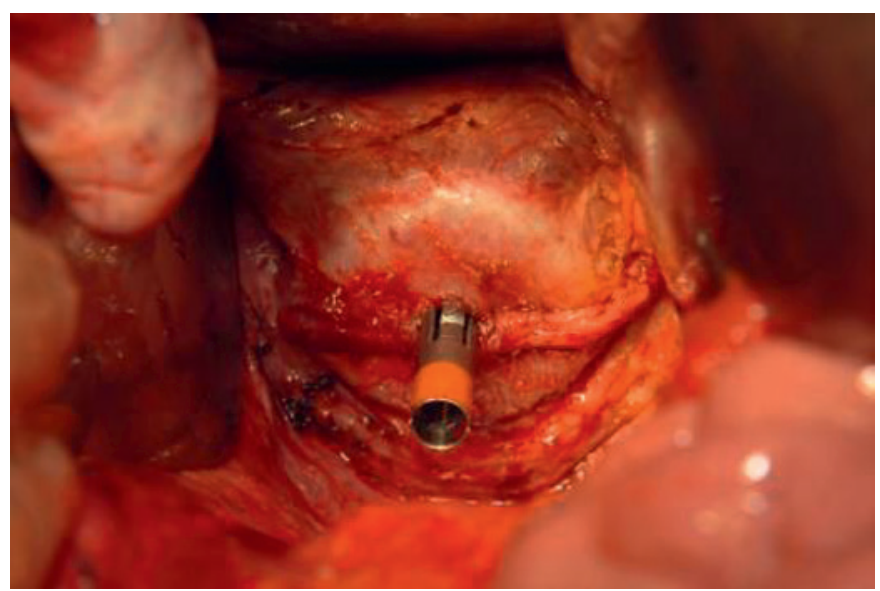

Fig. 3. Distal rectum ready for anastomosis.

cluding resection of the levatoric muscles, should be preferred instead of conventional conic APR (Fig. 4). Although there is a high rate of local, surgical site infections, patients' survival significantly increases when using this more radical approach $[20,21]$.

Patients presenting with stenosing tumors and acute ileus symptoms should receive a primary approach of a diverting stoma preferentially by laparoscopic technique in the sigma or distal ileum followed by staging, potential neoadjuvant therapy, and elective tumor surgery.

\section{Minimally Invasive Surgery}

The aim in almost all areas of surgery is to be highly effective with simultaneously causing as less tissue trau$\mathrm{ma}$ as possible to reduce negative side effects and improve patients' outcome. Since establishment of laparoscopic techniques, minimally invasive procedures have been increasingly introduced in all areas of oncologic surgery. Advantages compared to conventional techniques are faster mobilization after surgery due to lower pain rates and lower need of analgesic medication, especially opioids, reduced blood loss, shorter in-hospital stay, lower rates of cardiac and pulmonary complications, lower rates of surgical site infections, and lower rates of incisional hernias and intestinal obstruction [22]. For oncologic rectal resection, a clearly reduced 30 -day mortality rate has been shown $[23,24]$. However, similar to open surgery, surgical experience is important for results, complication rates, and oncologic outcome. Several studies (e.g., COLOR II trial) demonstrated similar long-term overall and disease-free survival rates for laparoscopic versus open rectal carcinoma resection $[2,24,25]$. The latest development of minimal invasive rectal surgery, robotic surgery, is described separately within this issue.

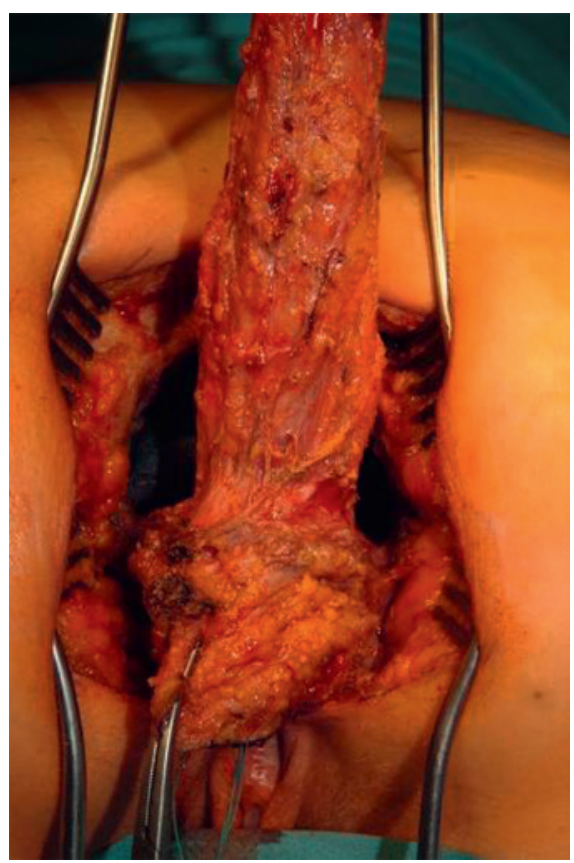

Fig. 4. Cylindric APR specimen.

Further innovation in minimally invasive rectal surgery is the concept of transanal TME (TATME). TATME is proposed in highly selected cases as an alternative approach or in addition to laparoscopic, robotic, or open dissection of the rectum via the abdominal approach (Fig. 5). The aim of this novel procedure is to overcome limitations and facilitate standard laparoscopic TME. A time-saving double team approach can be applied by combining transanal with transabdominal endoscopic techniques. First retrospective data seem to have lower rates of positive circumferential resection margins. The authors describe no higher complication rates compared to standard TME. However, results of randomized controlled trials and long-term survival rates of this technique are still not available [26].

\section{Neoadjuvant Therapy}

In deep and middle rectal carcinomas with $\mathrm{T} 3 / 4$ or $\mathrm{N}+$, a preoperative radiation with fractioned application of 45.0-50.4 Gy plus chemotherapy (intravenous or oral 5 -fluorouracil) is recommended in current international guidelines. This aims at a "downstaging" of the tumor to enable sphincter-preserving resections and at the reduction of local recurrence. After 6-8 weeks of recovery, elective operation should be performed. If the tumor size reduction is not in focus, a short-term radiation protocol with $5 \times 5$ Gy application scheme is possible followed by immediate operation within 10 days after the first radia- 


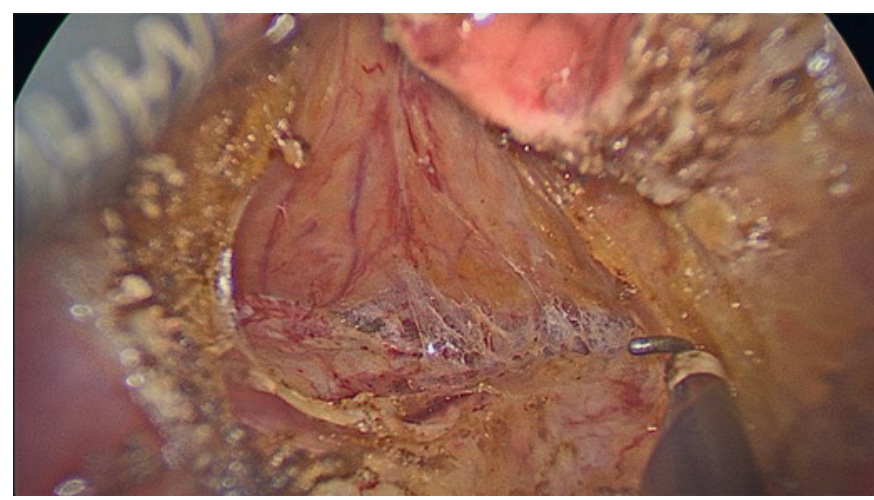

Fig. 5. Transanal TME.

tion. This also reduced the rate of local recurrence significantly, but in total, radiotherapy does not improve patient's overall survival [27]. The price for the lower rate of local recurrence is a higher perioperative morbidity (incontinence, sexual dysfunction, urinary incontinence, hip fractures, secondary tumors) and social restrictions [28]. Patients with T1/2 tumors and questionable lymph node metastasis as well as patients with $\mathrm{T} 3 \mathrm{a} / \mathrm{b}$ tumors of the middle rectum and limited perirectal infiltration in MRI (T3a $<1 \mathrm{~mm}$; T3b 1-5 mm) without suspicious lymph nodes or extramural vessel invasion can be treated by surgery primarily [2]. The circumferential resection margin from the mesorectal fascia measured in MRI should actually not be a decision-maker for direct surgery according to German S3 guidelines, but seems to be promising for future decision-making avoiding side effects of radiotherapy (MERCURY II trial) [29, 30]. Recently, a prospective multicenter observational study (OCUM Rectal Cancer Trial) could demonstrate that the restriction of neoadjuvant chemoradiotherapy only to high-risk patients achieves good results dependent on the MRI-measured distance of the tumor to the mesorectal fascia [31].

Rectal carcinomas of the upper third should be resected generally without any neoadjuvant treatment. In those cases, neoadjuvant therapy should only be applied in risk situations (T4, CRM+, clearly lymph node+) [2].

\section{Watch-and-Wait Policy versus Standard Resection}

TME significantly reduces the risk of local recurrence in rectal cancer patients. In combination with neoadjuvant radiotherapy or radiochemotherapy, the recurrence rate can be diminished and sphincter-preserving surgery can be increased in selected patients with lower locally advanced rectal cancer. The advances of a radical oncologic resection faces the risk of an up to $5 \%$ perioperative mortality and severe complications such as anastomotic leakage, sexual, bladder, and bowel dysfunction, or a permanent colostomy [32, 33].

More than a decade ago, reports came up that described a complete treatment response after neoadjuvant radiochemotherapy [34]. Those reports raised the question of an organ-preserving alternative to the established resectional rectal surgery after neoadjuvant treatment with an equivalent oncologic outcome. A recent metaanalysis showed an incidence of complete clinical response of $22.4 \%$ with a 3-year cumulative risk of local regrowth of $21.6 \%$ [35]. In almost $90 \%$ of these patients, salvage surgery was performed with a 3-year overall survival of $93.5 \%$. Thus, the authors conclude that a watchand-wait policy for patients with complete response following neoadjuvant radiochemotherapy appears feasible and safe without an increase in the risk of systematic disease or adverse survival outcomes.

As the effect of neoadjuvant radiation is obviously dependent on tumor stage, a potential benefit of neoadjuvant chemoradiation in early rectal cancers is under controversial discussion. Excellent oncological outcomes of TME surgery face the uncertain advances of watch-andwait strategies in cases of complete response after neoadjuvant therapy. In a retrospective analysis, the Sao Paulo group recently published $57 \%$ complete response rates for standard and even $86 \%$ for an extended chemoradiation regime on T2N0 rectal cancer patients [36]. However, this and other studies neglect the relevance of longterm side effects of chemoradiotherapy. It has been shown that long-term functional problems such as bowel or sexual disorders are significantly increased among patients having neoadjuvant chemoradiation compared with patients having surgery alone [28]. Underlying the aboveaverage rates of complete responses in the Brazilian data, a relevant number of patients still needs salvage surgery facing the above-mentioned side effects of surgery and chemoradiation. Therefore, and considering the promising results of studies such as the OCUM trial [31], we propose to rather curtail the frequency of neoadjuvant therapy than to expand the incidence to a larger group of patients. However, in patients who develop a complete response to neoadjuvant chemoradiation in locally advanced tumors, watch-and-wait seems to be a valid alternative, even though this notion has not be formally tested in randomized controlled trials.

\section{Adjuvant Therapy}

After surgery and receiving the final pathologic TNM stage, adjuvant therapy should be evaluated in interdisciplinary tumor boards. In case of T1/2 N0 rectal cancer, no adjuvant therapy is recommended. For patients with risk factors for local recurrence (R1, tumor perforation, poor 

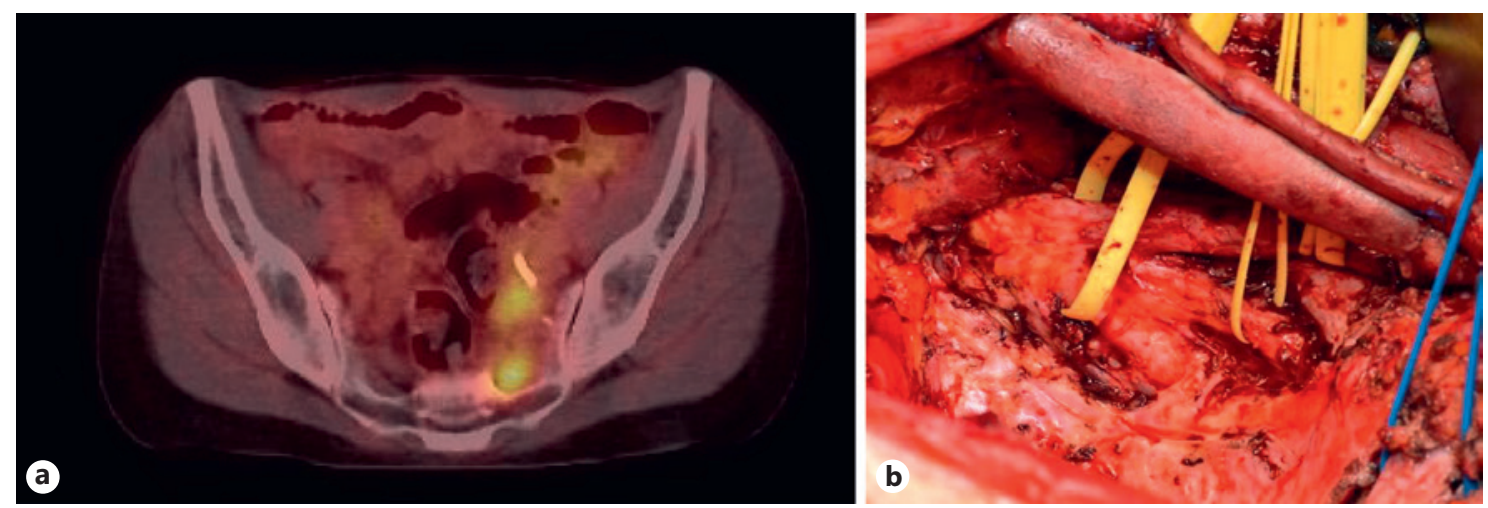

Fig. 6. a Local recurrence (PET scan). b Resection of local recurrence with preservation of pelvic plexus.

TME quality, pT4, pT3c/d, pN2, extranodal tumor spots in the mesorectum, pT3 in lower rectum), adjuvant chemotherapy within 8 weeks after surgery is standard therapy in Germany. However, a formal proof by randomized studies is still lacking. High rectal tumors should be treated analogous to colonic carcinomas [2].

\section{Therapy Concepts in Local Recurrence and Metastatic Disease}

\section{Local Recurrence}

The treatment of local recurrence depends on the therapy regimen given for primary tumor. In patients who did not receive neoadjuvant chemoradiation at initial treatment, the recurrent tumors should first undergo this therapy prior to surgery. In case of primary neoadjuvant radiation/chemotherapy and a possibility reaching $\mathrm{R} 0 \mathrm{re}$ section, direct surgery is recommended. In case of primary neoadjuvant-treated patients and borderline resectability, an individual concept between oncologist, radiotherapist, and surgeon is necessary that takes into account the intensity of previous therapy and the patients' history and current health situation. Surgical aspects should concentrate on reaching an R0 situation, which often can only be achieved by pelvic exenteration, including the removal of surrounding tissue (bladder, bone, vagina, uterus, nerves, vessels, etc.) (Fig. 6a, b). These operations are often followed by high rates of morbidity $(>30 \%)$ and low 30 -day mortality ( $2 \%)$, including loss of sexual, sphincter, and urinary function. Risk factors for worse outcome are positive resection margins and bone resection $[2,37$, 38].

\section{Peritoneal Carcinomatosis}

In case of limited peritoneal carcinomatosis without extra-abdominal metastasis, a complete cytoreductive surgery (removal of all visible tumor spots, complete tumor debulking) followed by hypertermic, intraperitoneal chemotherapy (HIPEC) can be performed. This leads to overall survival rates of $>50 \%$ in 5 years, which is superior to palliative chemotherapy. Patients benefit significantly more with a peritoneal carcinomatosis index (PCI) $<11$ than patients with PCI $<20$ [39-41]. Recent results of the Unicancer Prodige 7 trial, however, shed some doubt on this notion, as survival was not improved by HIPEC in this study.

\section{Liver/Lung Metastasis}

Patients in a metastatic situation should always be evaluated interdisciplinary in the presence of an experienced thoracic/liver surgeon, as surgery is the only curable therapy option. Limited metastatic disease can be cured by resection along the anatomic borders of lung and liver. If in doubt for resection, a "downsizing" with neoadjuvant chemotherapy using novel therapy regimens and antibodies can lead to secondary operability. To reduce postoperative liver failure (small-forsize-syndrome), new approaches are available which lead to a growth-allurement of the healthy part of the liver. The application of these techniques offers the possibility to resect initially unresectable liver metastases in curable intention. Those procedures like portal vein ligation, associating liver partition, and portal vein ligation for staged hepatectomy (ALPPS) only should be performed in high-volume centers. Cases with infiltration of surrounding tissues or big vessels in need for complex reconstructions must be evaluated individually taking into account age, fitness, medical history, and residual volume of liver/lung. To summarize, metastatic surgery is always a compromise between the technical possibilities and the will and personal situation of the patient. 


\section{Optimizing Perioperative Setting}

In times of aging patients, an optimal perioperative setting is inevitable in order to reduce patients' morbidity and mortality and to reduce costs. Before addressing the patient to surgery, a tumor-caused anemia due to deficiency of iron can be assessed easily and substituted reducing the perioperative transfusion of packed red blood cells (PRBC). The use of PRBC is associated with elevated morbidity and mortality [42]. Furthermore, the nutritional status of every patient should be evaluated by using a nutritional risk score (NRS). Dependent on this score, additional alimentation might lead to reduced perioperative complications due to hypoalbuminemia [43].

In times of fast-track surgery, preoperative mechanical bowel preparation before colorectal operations has been reduced to a minimum. Recent trials showed an advantage of mechanical bowel preparation and selective decontamination of the digestive tract in elective colorectal surgery to reduce collagenase-producing microbiome and thus, reduce the rate of anastomotic leakage and wound infections [44-46]. In our department, patients receiving elective rectal resection obtain mechanical bowel preparation and are treated with oral and rectal antibiotics perioperatively.
Furthermore, the World Health Organization recommends a number of perioperative procedures to reduce perioperative morbidity. Those include preoperative showering/bathing with (antibacterial) soap, reducing immunosuppressive medication, shaving hair by using hair clipping, preoperative antibiotic prophylaxis 30-60 min before surgery starts, keeping normothermia during operation, cleaning of the umbilicus, proper skin disinfection, use of stapling devices, proper change of gloves and instruments, and use of antibiotic-eluted suture material [47].

Modern cancer patient management furthermore includes psycho-oncological support as well as instruction and training in the use of the stoma care during hospital stay. Mobilization and respiratory muscle training should be standardized and performed under physiotherapeutic supervision as part of an "Enhanced Recovery After Surgery" (ERAS) program, which includes further measures.

\section{Disclosure Statement}

In reference to this review, all authors have no conflicts of interest to report.

\section{References}

1 2011/2012, K.i.D., Robert Koch-Institut (Hrsg) und die Gesellschaft der epidemiologischen Krebsregister in Deutschland e.V. (Hrsg). Berlin, 2015. 10. Ausgabe.

2 Onkologie L. (Deutsche Krebsgesellschaft, Deutsche Krebshilfe, AWMF): S3-Leitlinie Kolorektales Karzinom, Langversion 2.1, 2019, AWMF Registrierungsnummer: 021007OL, http://leitlinienprogrammonkologie. de/Leitlinien.7.0.html [Stand: Januar 2019].

3 Miles WE. A method of performing abdomino-perineal excision for carcinoma of the rectum and of the terminal portion of the pelvic colon (1908). CA Cancer J Clin. 1971 NovDec;21(6):361-4.

4 Ruo L, Guillem JG. Major 20th-century advancements in the management of rectal cancer. Dis Colon Rectum. 1999 May;42(5):56378.

5 Rao AR, Kagan AR, Chan PM, Gilbert HA, Nussbaum H, Hintz BL. Patterns of recurrence following curative resection alone for adenocarcinoma of the rectum and sigmoid colon. Cancer. 1981 Sep;48(6):1492-5.

6 Heald RJ, Husband EM, Ryall RD. The mesorectum in rectal cancer surgery-the clue to pelvic recurrence? Br J Surg. 1982 Oct;69(10): 613-6.

7 Heald RJ. The 'Holy Plane' of rectal surgery. J R Soc Med. 1988 Sep;81(9):503-8.
8 Heald RJ, Moran BJ, Brown G, Daniels IR. Optimal total mesorectal excision for rectal cancer is by dissection in front of Denonvilliers' fascia. Br J Surg. 2004 Jan;91(1):121-3.

9 Bernstein TE, Endreseth BH, Romundstad P, Wibe A; Norwegian Colorectal Cancer Group. Circumferential resection margin as a prognostic factor in rectal cancer. Br J Surg. 2009 Nov;96(11):1348-57.

10 MacFarlane JK, Ryall RD, Heald RJ. Mesorectal excision for rectal cancer. Lancet. 1993 Feb;341(8843):457-60.

11 Köckerling F, Reymond MA, Altendorf-Hofmann A, Dworak O, Hohenberger W. Influence of surgery on metachronous distant metastases and survival in rectal cancer. J Clin Oncol. 1998 Jan;16(1):324-9.

12 Bernhoff R, Martling A, Sjövall A, Granath F, Hohenberger W, Holm T. Improved survival after an educational project on colon cancer management in the county of Stockholm-a population based cohort study. Eur J Surg Oncol. 2015 Nov;41(11):1479-84.

13 den Dulk M, van de Velde CJ. Quality assurance in surgical oncology: the tale of the Dutch rectal cancer TME trial. J Surg Oncol. 2008 Jan;97(1):5-7.

14 Andreola S, Leo E, Belli F, Lavarino C, Bufalino R, Tomasic G, et al. Distal intramural spread in adenocarcinoma of the lower third of the rectum treated with total rectal resection and coloanal anastomosis. Dis Colon Rectum. 1997 Jan;40(1):25-9.
15 Kwok SP, Lau WY, Leung KL, Liew CT, Li AK. Prospective analysis of the distal margin of clearance in anterior resection for rectal carcinoma. Br J Surg. 1996 Jul;83(7):969-72.

16 Martin ST, Heneghan HM, Winter DC. Systematic review and meta-analysis of outcomes following pathological complete response to neoadjuvant chemoradiotherapy for rectal cancer. Br J Surg. 2012 Jul;99(7): 918-28.

17 Park JS, Park SY, Kim HJ, Cho SH, Kwak SG, Choi GS. Long-term Oncologic Outcomes After Neoadjuvant Chemoradiation Followed by Intersphincteric Resection With Coloanal Anastomosis for Locally Advanced Low Rectal Cancer. Dis Colon Rectum. 2019 Apr; 62(4):408-16

18 Hüttner FJ, Tenckhoff S, Jensen K, Uhlmann L, Kulu Y, Büchler MW, et al. Meta-analysis of reconstruction techniques after low anterior resection for rectal cancer. Br J Surg. 2015 Jun;102(7):735-45.

19 Choy PY, Bissett IP, Docherty JG, Parry BR, Merrie A, Fitzgerald A. Stapled versus handsewn methods for ileocolic anastomoses. Cochrane Database Syst Rev. 2011 Sep;(9): CD004320.

20 Holm T, Ljung A, Häggmark T, Jurell G, Lagergren J. Extended abdominoperineal resection with gluteus maximus flap reconstruction of the pelvic floor for rectal cancer. $\mathrm{Br}$ Surg. 2007 Feb;94(2):232-8. 
21 Huang A, Zhao H, Ling T, Quan Y, Zheng M, Feng B. Oncological superiority of extralevator abdominoperineal resection over conventional abdominoperineal resection: a metaanalysis. Int J Colorectal Dis. 2014 Mar;29(3): 321-7.

22 Rausa E, Bianco F, Kelly ME, Aiolfi A, Petrelli F, Bonitta G, et al. Systemic review and network meta-analysis comparing minimal surgical techniques for rectal cancer: quality of total mesorectum excision, pathological, surgical, and oncological outcomes. J Surg Oncol. 2019 Jun;119(7):987-98.

23 Gietelink L, Wouters MW, Bemelman WA, Dekker JW, Tollenaar RA, Tanis PJ; Dutch Surgical Colorectal Cancer Audit Group. Reduced 30-Day Mortality After Laparoscopic Colorectal Cancer Surgery: A Population Based Study From the Dutch Surgical Colorectal Audit (DSCA). Ann Surg. 2016 Jul; 264(1):135-40.

24 Bartels SA, Vlug MS, Hollmann MW, Dijkgraaf MG, Ubbink DT, Cense HA, et al.; Collaborative LAFA Study Group. Small bowel obstruction, incisional hernia and survival after laparoscopic and open colonic resection (LAFA study). Br J Surg. 2014 Aug;101(9): 1153-9.

25 Bonjer HJ, Deijen CL, Haglind E; COLOR II Study Group. A Randomized Trial of Laparoscopic versus Open Surgery for Rectal Cancer. N Engl J Med. 2015 Jul;373(2):194.

26 Arunachalam L, O’Grady H, Hunter IA, Killeen S. A Systematic Review of Outcomes After Transanal Mesorectal Resection for Rectal Cancer. Dis Colon Rectum. 2016 Apr; 59(4):340-50.

27 van Gijn W, Marijnen CA, Nagtegaal ID, Kranenbarg EM, Putter H, Wiggers T, et al.; Dutch Colorectal Cancer Group. Preoperative radiotherapy combined with total mesorectal excision for resectable rectal cancer: 12 year follow-up of the multicentre, randomised controlled TME trial. Lancet Oncol. 2011 Jun; 12(6):575-82.

28 Wibe A, Law WL, Fazio V, Delaney CP. Tailored rectal cancer treatment-a time for implementing contemporary prognostic factors? Colorectal Dis. 2013 Nov;15(11):133342.

29 Group MS; MERCURY Study Group. Diagnostic accuracy of preoperative magnetic resonance imaging in predicting curative resection of rectal cancer: prospective observational study. BMJ. 2006 Oct;333(7572):779.
30 Hermanek P, Hohenberger W, Fietkau R, Rödel C. Individualized magnetic resonance imaging-based neoadjuvant chemoradiation for middle and lower rectal carcinoma. Colorectal Dis. 2011 Jan;13(1):39-47.

31 Ruppert R, Junginger T, Ptok H, Strassburg J, Maurer CA, Brosi P, et al.; OCUM group. Oncological outcome after MRI-based selection for neoadjuvant chemoradiotherapy in the OCUM Rectal Cancer Trial. Br J Surg. 2018 Oct;105(11):1519-29.

32 Borowski DW, Bradburn DM, Mills SJ, Bharathan B, Wilson RG, Ratcliffe AA, et al.; Northern Region Colorectal Cancer Audit Group (NORCCAG). Volume-outcome analysis of colorectal cancer-related outcomes. $\mathrm{Br}$ J Surg. 2010 Sep;97(9):1416-30.

33 Paun BC, Cassie S, MacLean AR, Dixon E, Buie WD. Postoperative complications following surgery for rectal cancer. Ann Surg. 2010 May;251(5):807-18.

34 Marijnen CA. Organ preservation in rectal cancer: have all questions been answered? Lancet Oncol. 2015 Jan;16(1):e13-22.

35 Dattani M, Heald RJ, Goussous G, Broadhurst J, São Julião GP, Habr-Gama A, et al. Oncological and Survival Outcomes in Watch and Wait Patients With a Clinical Complete Response After Neoadjuvant Chemoradiotherapy for Rectal Cancer: A Systematic Review and Pooled Analysis. Ann Surg. 2018 Dec; 268(6):955-67.

36 Habr-Gama A, São Julião GP, Vailati BB, Sabbaga J, Aguilar PB, Fernandez LM, et al. Organ Preservation in cT2N0 Rectal Cancer After Neoadjuvant Chemoradiation Therapy: The Impact of Radiation Therapy Dose-escalation and Consolidation Chemotherapy. Ann Surg. 2019 Jan;269(1):102-7.

37 Rahbari NN, Ulrich AB, Bruckner T, Münter M, Nickles A, Contin P, et al. Surgery for locally recurrent rectal cancer in the era of total mesorectal excision: is there still a chance for cure? Ann Surg. 2011 Mar;253(3):522-33.

38 PelvEx Collaborative. Factors affecting outcomes following pelvic exenteration for locally recurrent rectal cancer. Br J Surg. 2018 May;105(6):650-7.

39 Elias D, Gilly F, Boutitie F, Quenet F, Bereder JM, Mansvelt B, et al. Peritoneal colorectal carcinomatosis treated with surgery and perioperative intraperitoneal chemotherapy: retrospective analysis of 523 patients from a multicentric French study. J Clin Oncol. 2010 Jan;28(1):63-8.
40 Goéré D, Malka D, Tzanis D, Gava V, Boige $\mathrm{V}$, Eveno $\mathrm{C}$, et al. Is there a possibility of a cure in patients with colorectal peritoneal carcinomatosis amenable to complete cytoreductive surgery and intraperitoneal chemotherapy? Ann Surg. 2013 Jun;257(6):1065-71.

41 Alzahrani NA, Valle SJ, Fisher OM, Sugarbaker PH, Yonemura Y, Glehen O, et al.; Peritoneal Surface Oncology Group International (PSOGI) and Big-RENAPE groups. Iterative cytoreductive surgery with or without hyperthermic intraperitoneal chemotherapy for colorectal peritoneal metastases: A multi-institutional experience. J Surg Oncol. 2019 Mar;119(3):336-46.

42 Amato A, Pescatori M. Perioperative blood transfusions for the recurrence of colorectal cancer. Cochrane Database Syst Rev. 2006 Jan;(1):CD005033.

43 Truong A, Hanna MH, Moghadamyeghaneh Z, Stamos MJ. Implications of preoperative hypoalbuminemia in colorectal surgery. World J Gastrointest Surg. 2016 May;8(5): 353-62.

44 Kiran RP, Murray AC, Chiuzan C, Estrada D, Forde K. Combined preoperative mechanical bowel preparation with oral antibiotics significantly reduces surgical site infection, anastomotic leak, and ileus after colorectal surgery. Ann Surg. 2015 Sep;262(3):416-25.

45 Scarborough JE, Mantyh CR, Sun Z, Migaly J. Combined Mechanical and Oral Antibiotic Bowel Preparation Reduces Incisional Surgical Site Infection and Anastomotic Leak Rates After Elective Colorectal Resection: An Analysis of Colectomy-Targeted ACS NSQIP. Ann Surg. 2015 Aug;262(2):331-7.

46 Abis GS, Stockmann HB, Bonjer HJ, van Veenendaal $\mathrm{N}$, van Doorn-Schepens $\mathrm{ML}$, Budding AE, et al.; SELECT trial study group. Randomized clinical trial of selective decontamination of the digestive tract in elective colorectal cancer surgery (SELECT trial). Br ) Surg. 2019 Mar; 106(4):355-63.

47 Allegranzi B, Zayed B, Bischoff P, Kubilay NZ, de Jonge S, de Vries F, et al.; WHO Guidelines Development Group. New WHO recommendations on intraoperative and postoperative measures for surgical site infection prevention: an evidence-based global perspective. Lancet Infect Dis. 2016 Dec;16(12):e288-303. 